\title{
Suppressing magnetic island growth by resonant magnetic perturbation
}

\author{
Q. Yu, S. Günter and K. Lackner \\ Max-Planck-Institut für Plasmaphysik, 85748 Garching, Germany
}

The effect of externally applied resonant magnetic perturbations (RMPs) on the growth of magnetic island is investigated based on two-fluid equations. It is found that if the local bi-normal electron fluid velocity at the resonant surface is sufficiently large, static RMPs of the same helicity and of moderate amplitude can suppress the growth of magnetic island in high temperature plasmas, which will otherwise grow, driven by an unfavorable plasma current density profile and bootstrap current perturbation. These results indicate that error field can be stabilizing for the island growth, if the error field amplitude is not too large and the local bi-normal electron fluid velocity is not too low, and that applied rotating RMPs with an appropriate frequency can be utilized to suppress the island growth in high temperature plasmas even for a low bi-normal electron fluid velocity. A significant change in the local equilibrium plasma current density gradient by small amplitude RMPs is found for realistic plasma parameters, being important for the island stability and expected to be more important for a fusion reactor with low plasma resistivity.

Key words: magnetic island, neoclassical tearing mode, resonant magnetic perturbation, two-fluid equations 


\section{Introduction}

It is well known that resonant magnetic perturbations (RMPs) have important effects on tokamak plasmas such as affecting magnetic islands inside the plasma [1-15], changing the plasma rotation velocity and density [4,16-20] and mitigating or suppressing edge localized modes (ELMs) [21-28].

As shown in tokamak experiments, externally applied RMPs of a sufficiently large amplitude can lock existing rotating magnetic islands or generate islands at the resonant surface even if the plasma is originally stable to tearing modes [1-11]. In some experiments, however, static RMPs are found to have a stabilizing effect on the tearing mode instabilities, and rotating magnetic islands have been stabilized by applying static RMPs of a moderate amplitude [1-4,9]. Theoretical results based on single-fluid equations have shown that RMPs can cause a nonuniform mode rotation due to the electromagnetic torque applied by RMPs. The island stays for a longer time in the half period when the RMP is stabilizing than in another half period when the RMP is destabilizing, and therefore there is a net stabilizing effect by RMPs $[4,9,12]$. Existing numerical studies on the stabilizing effect of RMPs on tearing mode instabilities, however, are limited to the single-fluid case applicable only for low electron temperature $[4,9,12]$. As neoclassical tearing modes (NTMs) can degrade plasma confinement or even lead to disruptions in existing tokamak discharges, the stabilization of NTMs is a very important issue for a fusion reactor. It is of great interest to investigate whether the stabilizing effect on tearing mode by RMPs also works for NTMs in high temperature plasmas, and to clarify the effect of the diamagnetic drift and the associated ion polarization current. Existing theories have already revealed the important role of diamagnetic drift and ion polarization current for the stability of a small magnetic island [29-35]. The island stability is of concern not only for NTMs but also for other MHD instabilities, since ideal lineal MHD instabilities can also trigger islands if the mode growth rate is slowed down in the nonlinear phase such that the plasma resistivity or other non- 
ideal effects play a role.

In this paper the effect of RMPs on the growth of an $\mathrm{m} / \mathrm{n}=2 / 1$ magnetic island is investigated numerically using the (reduced) two-fluid equations ( $\mathrm{m}$ and $\mathrm{n}$ being the poloidal and toroidal mode numbers). It is found that for a sufficiently large local bi-normal electron fluid velocity, static RMPs of the same helicity and of moderate amplitude can suppress the growth of magnetic island in high temperature plasmas.

\section{Numerical model}

The large aspect-ratio tokamak approximation is utilized. The magnetic field is defined as $\mathbf{B}=\mathrm{B}_{0 t} \mathbf{e}_{t}-\left(\mathrm{k}_{t} / \mathrm{k}_{\theta}\right) \mathrm{B}_{0 t} \mathbf{e}_{\theta}+\nabla \psi \times \mathbf{e}_{t}$, where $\psi$ is the helical flux function of the minor radius $\mathrm{r}$ and the helical angle, $\mathrm{k}_{\theta}=\mathrm{m} / \mathrm{r}$ and $\mathrm{k}_{\mathrm{t}}=\mathrm{n} / \mathrm{R}$ are the wave vector in $\mathbf{e}_{\theta}$ (poloidal) and $\mathbf{e}_{\mathrm{t}}$ (toroidal) direction, $\mathrm{R}$ is the major radius, and the subscript 0 denotes an equilibrium quantity. This definition implies that the equilibrium poloidal field is described by contributions from the last two terms in $\mathbf{B}$. The plasma velocity is given by $\mathbf{v}=\mathrm{v}_{\|} \mathbf{e}_{\|}+\nabla \phi \times \mathbf{e}_{\mathrm{t}}$, where $\phi$ is the stream function.

The two fluid equations used here include the mass conservation equation, the generalized Ohm's law, and the equation of motion in the perpendicular (after taking $\mathbf{e}_{t} \cdot \nabla \times$ ) and the parallel (to magnetic field) direction [36]. Normalizing the length to the minor radius a, the time $t$ to the resistive time $\tau_{R}=a^{2} \mu_{0} / \eta$, the helical flux $\psi$ to $\mathrm{aB}_{0 t}, \mathbf{v}$ to $a / \tau_{R}$, and the electron density $\mathrm{n}_{\mathrm{e}}$ to its value at the magnetic axis, these equations become [18]

$$
\begin{aligned}
& \frac{d n_{e}}{d t}=d_{1} \nabla_{\|} j-\nabla_{\|}\left(n_{e} v_{\|}\right)+\nabla_{\perp}\left(D_{\perp} \nabla_{\perp} n_{e}\right)+S_{n}, \\
& \frac{d \psi}{d t}=E_{0}-\eta_{\mathrm{N}}\left(j-j_{b}\right)-\frac{\eta_{\mathrm{N}} m_{e}}{n_{e} e^{2}} \frac{d j}{d t}+\Omega \nabla_{\|} n_{e}, \\
& \frac{d U}{d t}=S^{2} \nabla_{\|} j+\mu \nabla_{\perp}^{2} U+S_{m},
\end{aligned}
$$




$$
\frac{d v_{\|}}{d t}=-\mathrm{C}_{s}^{2} \nabla_{\|} P / n_{e}+\mu \nabla_{\perp}^{2} v_{\|},
$$

where $\mathrm{d} / \mathrm{dt}=\partial / \partial \mathrm{t}+\mathbf{v}_{\perp} \cdot \nabla_{\perp}, \mathrm{j}=-\nabla_{\perp}^{2} \psi$ is the parallel plasma current density, $\eta_{\mathrm{N}}$ the normalized resistivity, $\mathrm{j}_{\mathrm{b}}=-\mathrm{c}_{\mathrm{b}} \sqrt{ } \varepsilon\left(\partial \mathrm{p}_{\mathrm{e}} / \partial \mathrm{r}\right) / \mathrm{B}_{\mathrm{p}}$ the bootstrap current density, $\mathrm{c}_{\mathrm{b}}$ a constant of order of unity, $\varepsilon=\mathrm{r} / \mathrm{R}$ the inverse aspect ratio, $\mathrm{P}_{\mathrm{e}}=\mathrm{n}_{\mathrm{e}} \mathrm{T}_{\mathrm{e}}$ the electron pressure, $\mathrm{T}_{\mathrm{e}}$ the electron temperature, $\mathrm{B}_{\mathrm{p}}$ the poloidal magnetic field, and $m_{e}$ the electron mass. $E_{0}$ is the equilibrium electric field, $U=-\nabla_{\perp}^{2} \phi$ the plasma vorticity, $S_{\mathrm{n}}$ the particle source, and $\mathrm{S}_{\mathrm{m}}$ the poloidal momentum source leading to an equilibrium poloidal plasma rotation frequency $\omega_{\mathrm{E} 0} . \Omega=\beta \mathrm{d}_{1}, \mathrm{~d}_{1}=\omega_{\mathrm{ce}} / v_{\mathrm{e}}, \beta=4 \pi \mathrm{P}_{\mathrm{e}} / \mathrm{B}_{0 \mathrm{t}}{ }^{2}, \omega_{\mathrm{ce}}$ and $\nu_{\mathrm{e}}$ are the electron cyclotron and the collisional frequency, $S=\tau_{R} / \tau_{A}$, where $\tau_{A}=a / V_{A}$ is the toroidal Alfven time. $C_{S}$, $\mu$ and $\mathrm{D}_{\perp}$ are the normalized ion sound velocity, plasma viscosity, and perpendicular particle diffusivity. A constant electron temperature is assumed, and the cold ion assumption is made. The third term on the right hand side of equation (2) takes into account the electron inertia.

Equations (1)-(4) are solved simultaneously using the initial value code TM1, which had been used earlier for modelling the drift tearing mode instability and RMP-related physics problems [15,37]. The effect of RMP with $\mathrm{m} / \mathrm{n}=2 / 1$ is taken into account by the boundary condition

$$
\left.\psi_{2 / 1}\right|_{\mathrm{r}=\mathrm{a}}=\psi_{\mathrm{a}} \mathrm{aB} \mathrm{B}_{0 \mathrm{t}} \cos (\mathrm{m} \theta+\mathrm{n} \phi)
$$

where $\psi_{\mathrm{a}}$ describes the normalized $\mathrm{m} / \mathrm{n}=2 / 1$ helical magnetic flux amplitude at $\mathrm{r}=\mathrm{a}$. The radial magnetic field perturbation at $\mathrm{r}=\mathrm{a}$ is given by $\mathrm{b}_{1 \mathrm{r}}=-\mathrm{m} \psi_{\mathrm{a}} \mathrm{B}_{0 \mathrm{t}} \sin (\mathrm{m} \theta+\mathrm{n} \phi)$.

\section{Numerical results}

The radial profile of the original equilibrium plasma current density used for our calculations is of the form $\mathrm{j}_{\mathrm{j}} \mathrm{j}_{0}\left[1-(\mathrm{r} / \mathrm{a})^{2}\right]^{2}$, where $\mathrm{j}_{0}$ is the current density at $\mathrm{r}=0$. The corresponding radial profile of the equilibrium safety factor $\mathrm{q}$ is monotonic with the $\mathrm{q}=2$ surface located at $\mathrm{r}_{2 / 1}=0.628 \mathrm{a}$. The $\mathrm{m} / \mathrm{n}=2 / 1$ classical tearing mode is unstable with this q-profile, and the magnetic 
island grows to a width of $0.2 \mathrm{a}$ in the single fluid calculation for $\psi_{\mathrm{a}}=0$, even when the bootstrap current perturbation is neglected.

The input parameters are based on ASDEX Upgrade experimental parameters. The toroidal magnetic field is $\mathrm{B}_{0 t}=2 \mathrm{~T}$, the plasma minor and major radius are $\mathrm{a}=0.5 \mathrm{~m}$ and $\mathrm{R}=1.7 \mathrm{~m}$ (plasma current $\mathrm{I}_{\mathrm{p}}=0.37 \mathrm{MA}$ in cylinder geometry), $\mathrm{T}_{\mathrm{e}}=2 \mathrm{keV}$, and $\mathrm{n}_{\mathrm{e}}=3 \times 10^{19} \mathrm{~m}^{-3}$. These parameters lead to $\mathrm{S}=2.6 \times 10^{8}, \mathrm{C}_{\mathrm{s}}=2 \times 10^{7}\left(\mathrm{a} / \tau_{\mathrm{R}}\right), \eta_{\mathrm{N}}=1$, and $\mathrm{d}_{1}=3.1 \times 10^{7}$. A parabolic profile for the electron density is taken, leading to an electron diamagnetic drift frequency $\omega *_{\mathrm{e} 0}=1.5 \times 10^{5} / \tau_{\mathrm{R}}\left(\mathrm{f}_{*_{\mathrm{e}} 0}=3.18 \mathrm{kHz}\right)$ with $\Omega=6.1 \times 10^{4} / \tau_{\mathrm{R}}$. Assuming that the plasma momentum transport is at an anomalous transport level of $0.2 \mathrm{~m}^{2} / \mathrm{s}$, one finds $\mu=18.8\left(\mathrm{a}^{2} / \tau_{\mathrm{R}}\right)$ in normalized units. The perpendicular particle diffusivity is assumed to be $D_{\perp}=\mu / 5$. These parameters are used as input for our calculations except when specified otherwise.

In tokamak experiments the plasma rotation is essentially toroidal [4], while in Eqs. (1)-(4) due to the large aspect ratio approximation only the poloidal rotation is included, so that a larger plasma viscosity for the $\mathrm{m} / \mathrm{n}=0 / 0$ component, $\mu_{0 / 0}=18.8 \times 10^{2}\left(\mathrm{a}^{2} / \tau_{\mathrm{R}}\right)$, is used in calculations, if not explicitly specified. This value guarantees a reasonable balance between the electromagnetic and viscous force, based on the following considerations [4]: (a) The electromagnetic force in the toroidal direction is smaller by a factor $(\mathrm{n} / \mathrm{m})\left(\mathrm{r}_{\mathrm{s}} / \mathrm{R}\right)$ than that in the poloidal direction. (b) To have the same mode frequency due to the plasma rotation, the toroidal rotation velocity should be $(\mathrm{m} / \mathrm{n})\left(\mathrm{R} / \mathrm{r}_{\mathrm{s}}\right)$ times larger than the poloidal one. These two effects lead to a larger viscous force compared to the electromagnetic force for the toroidal rotation case by a factor $\left[(\mathrm{m} / \mathrm{n})\left(\mathrm{R} / \mathrm{r}_{\mathrm{s}}\right)\right]^{2}$, which is of the order $10^{2}$. It will be shown in the following that the results are essentially not changed without the factor $10^{2}$ for $\mu_{0 / 0}$ when the island width is small.

When the equilibrium plasma rotation frequency $\omega_{\mathrm{E} 0}$ is taken to be zero, the time evolution of the island width, obtained by directly solving equations (1)-(4), are shown in Fig. 1 (left) for four different cases, with the local bootstrap current density fraction $\mathrm{f}_{\mathrm{b}}=0.35$ at $r_{2 / l}$. The island 
width is calculated from $W=4\left[\psi_{2 / 1} /\left(B_{\theta} q / q^{\prime}\right)\right]^{1 / 2}$ at $r_{2 / 1}$, where $\psi_{2 / 1}$ is the perturbed flux of the $\mathrm{m} / \mathrm{n}=2 / 1$ component. The blue, red and green curves in figure 1 correspond to $\psi_{\mathrm{a}}=0,10^{-4}$ and $1.2 \times 10^{-4}$, respectively. It is seen that the island grows without applying the RMP or for a too large RMP amplitude, $\psi_{\mathrm{a}}=1.2 \times 10^{-4}$. For a moderate RMP amplitude, e.g. $\psi_{\mathrm{a}}=10^{-4}$, however, the island width is suppressed to a low value, being smaller than 0.01 a, i.e. comparable to $\rho_{\mathrm{s}}$, the ion Larmor radius by using electron temperature. The black curve corresponds to the single fluid case obtained with $\psi_{\mathrm{a}}=0$ and $\Omega=\mathrm{d}_{1}=\mathrm{C}_{\mathrm{s}}=\mathrm{m}_{\mathrm{e}}=0$, for which the island grows and eventually saturates at a width about $0.2 \mathrm{a}$ in a time scale $0.03 \tau_{\mathrm{R}}$.
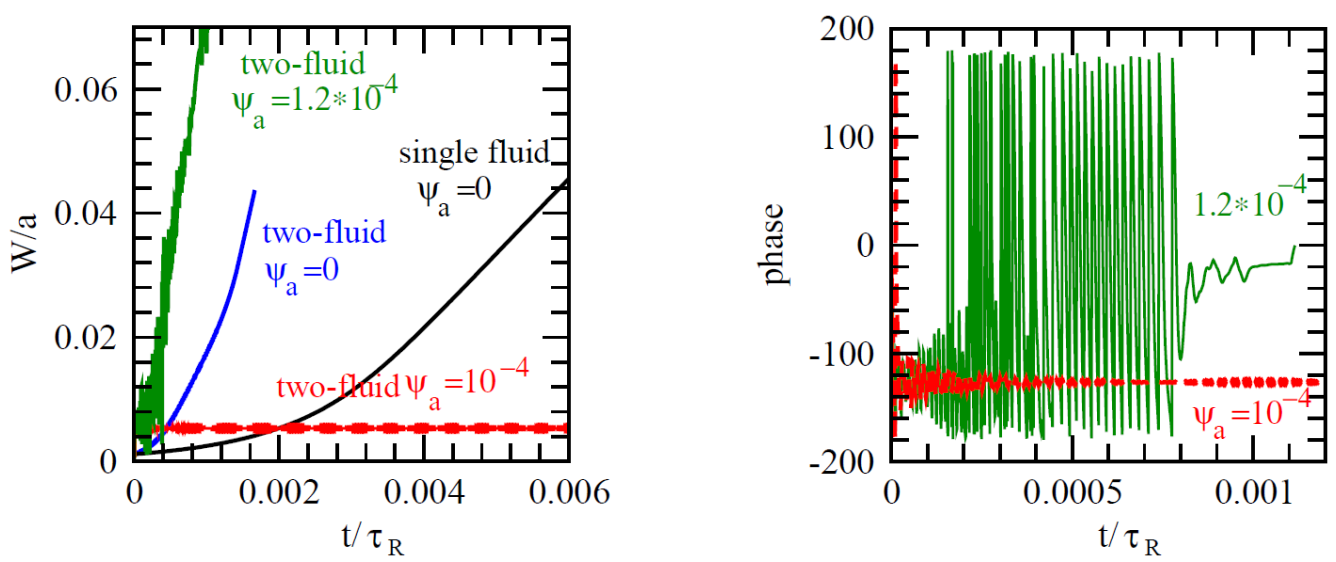

Figure 1 (left) Time evolution of the island width with $f_{b}=0.35$ and $\omega_{E 0}=0$ for the single fluid case (with $\psi_{a}=0$ ) and two-fluid cases (with $\psi_{a}=0,10^{-4}$, and $1.2 \times 10^{-4}$ ). For a moderate RMP amplitude, $\psi_{a}=10^{-4}$, the island growth is suppressed in the two-fluid calculation. (right) Corresponding time evolution of the phase of island's o-point for two-fluid cases with $\psi_{a}=10^{-4}$ and $1.2 \times 10^{-4}$. For $\psi_{a}=10^{-4}$, the island is locked at $-127^{\circ}$ (The phase of $R M P$ is at $0^{\circ}$ ).

The time evolution of the phase of the island's o-point is shown in Figure 1 (right) for twofluid cases with $\psi_{\mathrm{a}}=10^{-4}$ and $1.2 \times 10^{-4}$. For the large RMP amplitude $\left(\psi_{\mathrm{a}}=1.2 \times 10^{-4}\right)$, the island is locked to the phase of the RMP at $0^{\circ}$ when the island width is sufficiently large. For $\psi_{\mathrm{a}}=10^{-4}$, the island's o-point is locked to a phase $-127^{\circ}$. Such a relative phase between the island's o-point and the RMP, $\Delta \varphi=-127^{\circ}$, is unfavorable for the island growth, and thus the island is suppressed by the 
RMP. There is a range in the RMP amplitude for island suppression. With the input parameters of figure 1 , this range exists for $0.5 \times 10^{-4}<\psi_{\mathrm{a}}<1.1 \times 10^{-4}$ (corresponding to the radial magnetic field from 0.1 to $0.22 \mathrm{mT}$ at $\mathrm{r}=\mathrm{a}$, being comparable to that typically used in RMP experiments [28]). If the RMP amplitude is too small, in the early nonlinear phase the island grows slower than that without applying RMPs. If the RMP amplitude is too large to exceed this range, it will cause locked mode at $0^{\circ}$.

To understand the cause of the stabilizing effect of RMPs, calculations have been carried out with other input parameters. The mode stabilization by RMPs has also been found for the classical tearing mode $\left(\mathrm{f}_{\mathrm{b}}=0\right)$, neglecting the electron inertia in Ohm's law or taking the ion sound velocity to be zero. The stabilizing effect of RMPs is however strongly affected by the diamagnetic drift frequency. Decreasing the equilibrium electron diamagnetic drift frequency by using a smaller value of $\Omega$ in equation (2), the stabilizing effect of RMPs decreases and eventually disappears when the value of $\Omega$ is reduced by about one order of magnitude. With increasing value of $S$ or decreasing values of the perpendicular particle diffusivity, the suppression of island growth by the RMP has been found for smaller RMP amplitudes. A large value of plasma viscosity is not required for the stabilizing effect. The island growth is still suppressed if the value of plasma viscosity is decreased by more than two orders of magnitude, e.g. for $\psi_{\mathrm{a}}=6 \times 10^{-5}$. Using the single fluid equations with an equilibrium plasma rotation frequency being about the same as the electron diamagnetic drift frequency of the two-fluid case, however, no island suppression is found except for unrealistically large plasma viscosities, in agreement with the analytical finding that plasma viscosity is stabilizing [35]. The mode stabilization by RMPs is thus much stronger in two-fluid case with a strong influence of electron diamagnetic drift frequency. 


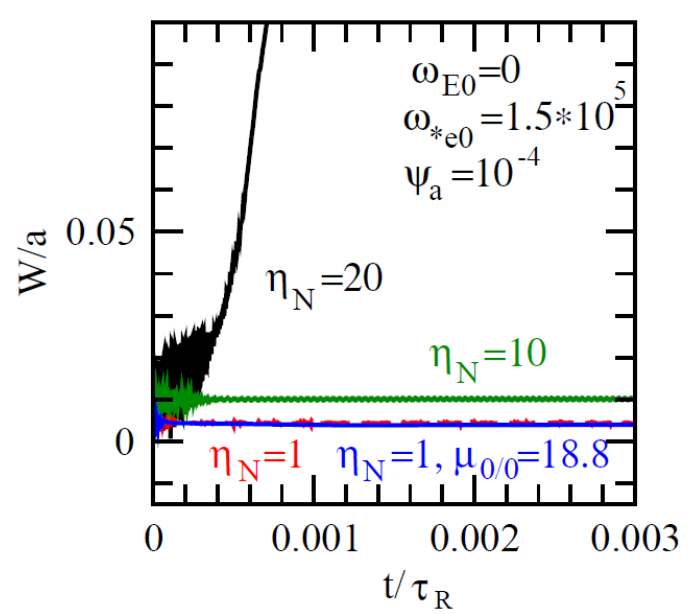

Figure 2 Time evolution of the island width with $\psi_{a}=10^{-4}, f_{b}=0$ and $\omega_{E 0}=0$ for $\eta_{N}=20,10$ and 1 , respectively. The island grows only for large plasma resistivity. The island is also suppressed for reduced viscosity $\mu_{0 / 0}=18.8\left(a^{2} / \tau_{R}\right)$ at low resistivity $\eta_{N}=1$ (blue curve).

If the plasma resistivity is sufficiently large, the mode stabilization by RMPs disappears. The time evolution of the island width is shown in Fig. 2 with $\psi_{\mathrm{a}}=10^{-4}, \mathrm{f}_{\mathrm{b}}=0$ and $\omega_{\mathrm{E} 0}=0$. The black, green and red curves are obtained for $\eta_{N}=20,10$ and 1, respectively, keeping the other input parameters unchanged. The island grows only for the largest plasma resistivity but is suppressed for smaller ones. The blue curve in figure 2 is obtained for a small plasma viscosity $\left(\mu_{0 / 0}=18.8\right.$ $\left(\mathrm{a}^{2} / \tau_{\mathrm{R}}\right)$, i.e. without the increase of $\mu_{0 / 0}$ by a factor $\left.10^{2}\right)$ and low resistivity $\left(\eta_{\mathrm{N}}=1\right)$, and in this case the island growth is also suppressed. It has been found that the value of the plasma viscosity $\mu_{0 / 0}$ is important only for a sufficiently large RMP amplitude and/or island width.

It should be mentioned that, although the equilibrium plasma rotation velocity is taken to be zero for the results shown in figures 1 and 2, RMPs always drive a $\mathrm{m} / \mathrm{n}=0 / 0$ component plasma rotation towards the ion drift direction to decrease the local bi-normal electron velocity at $r=r_{2 / 1}$, as found before [19]. The driven rotation velocity is larger for a smaller values of $\mu_{0 / 0}$. The equilibrium electron rotation frequency at $r=r_{2 / 1}$ can be defined as $\omega_{e} \perp \equiv\left(1-\omega_{0}\right) \omega * 0$ to include the contributions from both the plasma rotation (electric drift) and electron diamagnetic drift frequency, where $\omega_{0} \equiv-\omega_{\mathrm{E} 0} / \omega *_{\mathrm{e} 0}$. 


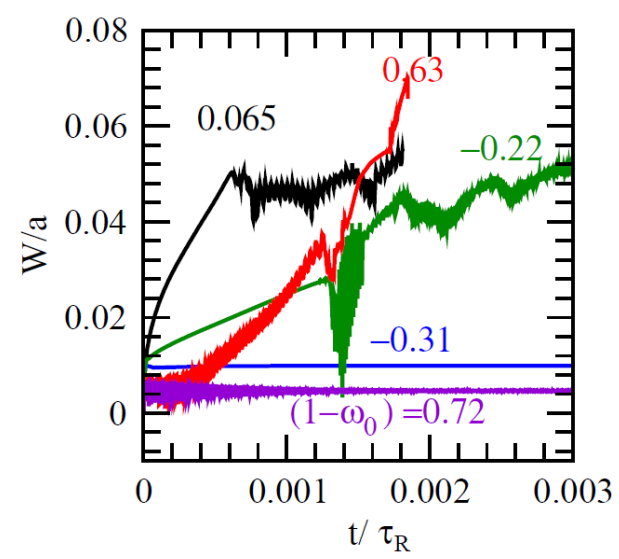

Figure 3 Time evolution of the island width for $\omega_{e \perp} / \omega_{*_{e} 0}=0.72,0.63,0.065,-0.22$, and -0.31 with $\psi_{a}=6 \times 10^{-5}$ and $f_{b}=0.35$. The island grows for a sufficiently small $\left|\omega_{e \perp}\right|$ but is suppressed for a large one.

For different equilibrium plasma rotation velocities, the time evolution of the island width is shown in Fig. 3 with $\psi_{\mathrm{a}}=6 \times 10^{-5}$ and $\mathrm{f}_{\mathrm{b}}=0.35$ for $\omega_{0}=0.28,0.37,0.935,1.22$ and 1.31, corresponding to $\omega_{\mathrm{e} \perp} / \omega_{\mathrm{e}_{0}}=0.72,0.63,0.065,-0.22$, and -0.31 , as marked in the figure. The positive (negative) values of $\omega_{\mathrm{e} \perp} / \omega * \mathrm{e} 0$ correspond to plasma rotation frequencies being smaller (larger) than $\left|\omega *_{\mathrm{e}}\right| \mid$. The island grows for a sufficiently small value of $\left|\omega_{\mathrm{e} \perp}\right|$, but is suppressed for large ones. It is interesting to note that the island suppression by RMPs is asymmetric on the two sides of $\omega_{\mathrm{e} \perp}=0$, being more effective for $\omega_{\mathrm{e} \perp}<0$, i.e., the plasma rotation in the ion diamagnetic drift direction with a frequency being lager than $|\omega * \mathrm{e} 0|$. This asymmetry can be explained by the partial flattening of the electron density profile and the associated reduction of the electron diamagnetic drift frequency, and the total bi-normal electron fluid velocity increases compared to the unperturbed one for finite island size with $\omega_{\mathrm{e} \perp}<0$. Such an asymmetry was also found in field penetration experiments [8]. For the cases with $\left|\omega_{\mathrm{e} \perp} / \omega *_{\mathrm{e}}\right|>1$, island growth suppression by RMPs is also found, and the range in the required RMP amplitude for suppressing island growth is larger with increasing $\left|\omega_{\mathrm{e} \perp} / \omega *_{\mathrm{e} 0}\right|$. 

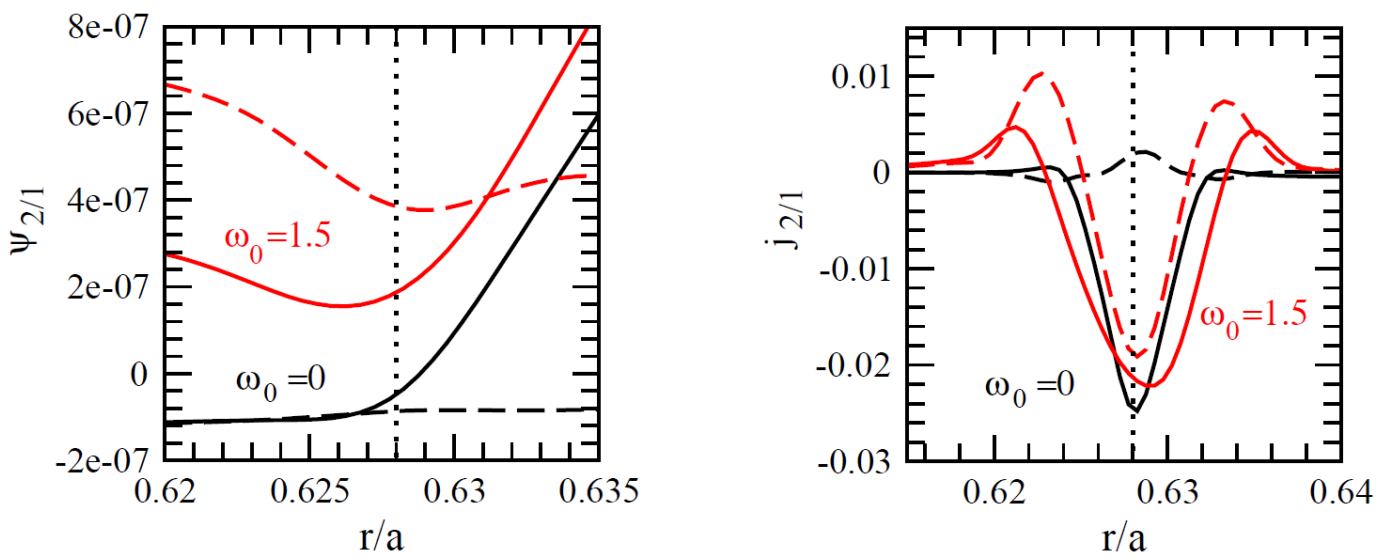

Figure 4 (left) Radial profiles of the (normalized) perturbed flux of the $m / n=2 / 1$ component, $\psi_{2 / 1}$, in steady state with $f_{b}=0.35$ and $\psi_{a}=6 \times 10^{-5}$ for $\omega_{0}=0$ (black curves) and 1.5 (red), where the solid (dashed) curves show the real (imaginary) part. The phase of the applied RMPs is at $0^{\circ}$. (right) Corresponding radial profiles of the (normalized) $m / n=2 / 1$ component plasma current density, $j_{2 / 1}$.

Radial profiles of the (normalized) perturbed flux of the $\mathrm{m} / \mathrm{n}=2 / 1$ component, $\psi_{2} / 1$, in steady state with $\mathrm{f}_{\mathrm{b}}=0.35$ and $\psi_{\mathrm{a}}=6 \times 10^{-5}$ are shown in Fig. 4 (left) for $\omega_{0}=0$ (black curves) and 1.5 (red), where the solid (dashed) curves are for the real (imaginary) part. For these two cases the island growth is suppressed by RMPs. The real part of $\psi_{2 / 1}$ at $r_{2 / 1}$ is negative for $\omega_{0}=0\left(\Delta \varphi=-127^{\circ}\right)$ but is positive for $\omega_{0}=1.5\left(\Delta \varphi=69.2^{\circ}\right)$. Corresponding radial profiles of the (normalized) $\mathrm{m} / \mathrm{n}=2 / 1$ component plasma current density, $j_{2 / 1}$, are shown in Fig. 4 (right). The real parts of $j_{2 / 1}$ are in the same direction for both cases, since the shielding current density is always in the direction to cancel the externally applied RMP. 


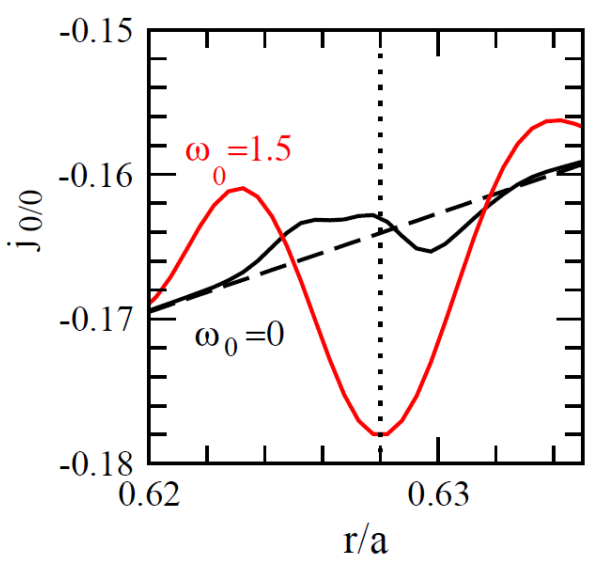

Figure 5 Corresponding to figure 4, radial profiles of the (normalized) $m / n=0 / 0$ component of the plasma current density, $j_{0 / 0}$, in steady state (solid curves). The dashed curve is the original equilibrium plasma current density, being negative in our calculations.

Corresponding to figure 4 , the radial profiles of the (normalized) $m / n=0 / 0$ component of the plasma current density, jo/0, in steady state are shown in Fig. 5 by the solid curves. The dashed curve is the original equilibrium plasma current density, taking to be negative in our calculations. The change of the current density is due to the nonlinear terms in Ohm's law, being particularly important for low plasma resistivity $\left(\eta_{N}=1\right)$ in high temperature plasmas. When using the jo/0 profile of the case $\omega_{0}=1.5$ as the equilibrium current density for linear calculations (without applying RMPs), the $2 / 1$ mode is found to be linearly stable, indicating that the nonlinear change in the $\mathrm{m} / \mathrm{n}=0 / 0$ component plasma current density profile plays a stabilizing role in this case. However, when using the $\mathrm{j}_{0 / 0}$ profile for the case $\omega_{0}=0$ for linear calculations (without applying RMPs), the tearing mode is found to grow at an even larger linear growth rate compared to the case of using the original equilibrium plasma current density. The significant change in the local $\mathrm{m} / \mathrm{n}=0 / 0$ plasma current density gradient by small amplitude RMPs for high temperature plasmas is important for the island stability, and it can be either stabilizing or destabilizing depending on the plasma rotation velocity. Such an effect is expected to be more important for a fusion reactor with low plasma resistivity. 


\section{Discussion and summary}

It is already known that the diamagnetic drift and the associated ion polarization current are important for the stability of small magnetic islands [29-34,37]. Both effects have been included in our calculations. The ion polarization current is particularly important for significant equilibrium plasma rotation. This can be explained by looking at the modified Rutherford equation, in which the ion polarization current term is proportional to $-\left(\omega-\omega_{E}\right)\left(\omega-\omega_{E}-\omega * *_{e}\right) / W^{3}[29-$ 34]. For vanishing mode frequency $\omega$ and $\omega_{\mathrm{E}}$, this effect is small, suggesting that in these cases the suppression of island growth by RMPs is due to the looking of the small island in the stabilizing phase of the RMP, i.e. $90^{\circ}<\Delta \varphi<270^{\circ}$. In the case of significant plasma rotation into the ion drift direction with a frequency being larger than electron diamagnetic drift frequency, i.e. $\omega_{\mathrm{e} \perp} / \omega_{\mathrm{e} 0}<0$ or $\omega_{0}>1$, the island's o-point is locked in the destabilizing phase of the RMP as shown in figure 4 (i.e. $\Delta \varphi<90^{\circ}$ ). In this case the ion polarization current is stabilizing. For small locked island, the ion polarization current term in the modified Rutherford equation is proportional to $\left.\omega_{\mathrm{E}}\left(\omega_{\mathrm{E}}+\omega *_{\mathrm{e}}\right)\right) / \mathrm{W}^{3}$. In addition, in such cases the modification of the $\mathrm{m} / \mathrm{n}=0 / 0$ component of the plasma current density by RMPs is stabilizing. By including this effect as well as electron inertia, our calculations go beyond existing analytical theory. For direct comparison with experiments, toroidal geometry and shaping have to be taken into account.

In summary, the effect of an externally applied RMP of the same helicity on the growth of NTMs is investigated based on two fluid equations. If the absolute value of local electron fluid velocity at the resonant surface is sufficiently large, the magnetic island growth is found to be suppressed by RMPs of moderate amplitude even with a significant fraction of local bootstrap current density. These results suggest that error field can be stabilizing for the NTM's onset, if the error field amplitude is not too large and the local bi-normal electron fluid velocity is not too low, and that applied rotating RMPs with an appropriate frequency can be utilized to stabilize magnetic 
islands in high temperature plasmas even for a low bi-normal electron fluid velocity. A significant change in the local equilibrium plasma current density gradient by small amplitude RMPs is found for realistic plasma parameters, being important for the island stability and expected to be more important for a fusion reactor with low plasma resistivity. 


\section{References}

[1] Karger F. et al 1975 Plasma physics and controlled nuclear fusion research 1974 Proc. 5th Int. Conf. (Tokyo, 1974) vol 1 (Vienna: IAEA) p 207 (www-naweb.iaea.org/napc/physics/

FEC/STIPUB381 VOL1.pdf)

[2] Zhao Q.C. et al 1985 Plasma physics and controlled nuclear fusion research 1984 Proc. 10th Int. Conf. (London, 1984) vol 1 (Vienna: IAEA) p 345

(wwwnaweb.iaea.org/napc/physics/FEC/STIPUB670 VOL1.pdf)

[3] Ellis J.J., Howling A.A., Morris A.W. and Robinson D.C. 1985 Plasma physics and controlled nuclear fusion research 1984 Proc. 10th Int. Conf. (London, 1984) vol 1 (Vienna: IAEA) p 363 (www-naweb.iaea.org/napc/physics/FEC/STIPUB670 VOL1.pdf)

[4] Hender T.C. et al 1992 Nucl. Fusion 32, 2091

[5] Buttery R.J. et al 2000 Nucl. Fusion 40, 807

[6] La Haye R.J., Fitzpatrick R., Hender T.C. et al 1992 Phys. Fluids B 4, 2098

[7] Wofle S.W., Hutchinson I.H., Granetz R.S. et al 2005 Phys. Plasmas 12, 056110

[8] Koslowski H.R., Liang Y., Krämer-Flecken A. et al 2006 Nucl. Fusion 46, L1

[9] Hu Q., Yu Q., Rao B., Ding Y. H. et al 2012 Nucl. Fusion 52, 083011

[10] Nave M.F.F. and Wesson J.A. 1990 Nucl. Fusion 30, 2575

[11] Zohm H., Kallenbach A., Bruhns H. et al 1990 Europhys. Lett. 11, 745

[12] Fitzpatrick R. 1993 Nucl. Fusion 33, 1049

[13] Waelbroeck F. L. 2003 Phys. Plasmas 10, 4040

[14] Yu Q., Günter S., Kikuchi Y. and Finken K.H. 2008 Nucl. Fusion 48, 024007

[15] Yu Q. and Günter S 2008 Nucl. Fusion 48, 065004

[16] Finken K.H., Abdullaev S.S., De Bock M.F.M. et al 2005 Phys. Rev. Lett, 94, 015003

[17] Finken K.H., Abdullaev S.S., Jakubowski M.W. et al 2007 Phys. Rev. Lett, 98, 065001

[18] Yu Q. and Günter S. 2009 Nucl. Fusion 49062001

[19] Yu Q., Günter S. and Finken K.H. 2009 Phys. Plasmas 16, 042301

[20] Yu Q. and Günter S. 2011 Nucl. Fusion 51073030

[21] Evans T.E., Moyer R.A., Thomas P.R. et al 2004 Phys. Rev. Lett, 92, 235003

[22] Liang Y. et al 2007 Phys. Rev. Lett 98265004

[23] Suttrop W. et al 2011 Phys. Rev. Lett 106225004

[24] Shoji T., Tamai H., Miura Y., Mori M. and Ogawa H. 1992 J. Nucl. Mater. 196 296-300

[25] Kirk A. et al, MAST and ASDEX Upgrade Teams 2015 Nucl. Fusion 55043011

[26] Evans T. E., Fenstermacher M. E., Moyer R.A. et al 2008 Nucl. Fusion 48024002

[27] Jeon Y. M. et al 2012 Phys. Rev. Lett. 109035004

[28] Suttrop W. et al 2017 Plasma Phys. Control. Fusion 59014049

[29] Smolyakov A.I. 1993 Plasma Phys. Control. Fusion 35657

[30] Wilson H.R., Connor J.W., Hastie R.J. and Hegna C.C. 1996 Phys. Plasmas 3248 
[31] Connor J.W., Waelbroeck F.L. and Wilson H.R. 2001 Phys. Plasmas 82835

[32] Sauter O. et al 1997 Phys. Plasmas 41654

[33]Waelbroeck F.L., Connor J.W. and Wilson H.R. 2001 Phys. Rev. Lett. 87215003

[34]Fitzpatrick R., Waelbroeck F.L. and Militello F. 2006 Phys. Plasmas 13122507

[35] Waelbroeck F.L. and Fitzpatrick R. 1997 Phys. Rev. Lett. 781703

[36] Hazeltine R. D. et al 1985 Phys Fluids 282466.

[37] Yu Q. 2010 Nucl. Fusion 50025014 